\title{
TEO: Uma suíte de jogos interativos para apoio ao tratamento de crianças com autismo
}

\author{
Douglas L. L. Moura ${ }^{1}$, Diogenes L. S. O. Filho ${ }^{1}$, Anny J. G. Silva ${ }^{1}$, \\ Pedro V. V. Paiva ${ }^{1}$, Thiago B. M. Sales ${ }^{1}$, Rodolfo C. Cavalcante ${ }^{1}$, \\ Fabiane S. Queiroz ${ }^{1}$ \\ ${ }^{1}$ Universidade Federal de Alagoas - Campus Arapiraca (UFAL) \\ Caixa Postal 57309-005 - Arapiraca - AL - Brasil \\ \{douglasllmoura, diogeneslaertiussof, anny.jgs\}@gmail.com, \\ \{pedro.paiva@arapiraca.ufal.br, thiagobrunoms\}egmail.com, \\ \{rodolfo.cavalcante, fabiane.queiroz\}earapiraca.ufal.br
}

\begin{abstract}
The Autistic Spectrum Disorder (ASD (Autistic Spectrum Disorder) is defined as a set of complex disorders that affect the brain and accompany the individual throughout the life. Through the consolidation of current technologies in support of setting treatment and focus on education in the autistic universe, we propose an evaluation and a discussion of a methodology for the environment of interactive games called TEO (from portguese, Tratar, Estimular e Orientar - or Treat, Encourage and Guide). The purpose is, therefore, to aid the treatment of authism in a transdisciplinary way, articulating through interactive games the development of logical reasoning, visual perception, concentration and other general skills.
\end{abstract}

Resumo. O Transtorno do Espectro Autista (TEA) é definido como um conjunto de desordens complexas que afetam o cérebro e acompanham o indivíduo por toda a vida. Com a consolidação das tecnologias atuais no cenário de suporte ao tratamento e educação com foco no universo autista, propõe-se no presente artigo uma avaliação e uma discussão acerca da metodologia para o ambiente de jogos interativos denominado TEO (Tratar, Estimular e Orientar). Objetiva-se, desta forma, fundamentar os processos que resultaram na criação de um ambiente que auxilie no tratamento do autismo de maneira transdisciplinar, articulando através de jogos interativos o fomento do raciocínio lógico, da percepção visual, da concentração e de outras habilidades de modo geral.

\section{Introdução}

Em 1943, Leo Kanner [Kanner 1943] descreveu o Transtorno do Espectro Autistas (TEA), ou simplesmente autismo, como uma síndrome específica, caraterizada por sua precocidade, sintomatologia, evolução e pelas perturbações das relações afetivas com o meio. Dentre os muitos sintomas detectados em pacientes diagnosticados com autismos, estão:

- Déficit de coerência central: Visto como dificuldade em manter a atenção em informações, atendo-se somente a detalhes específicos de gosto próprio [Joseph et al. 2009];

- Foco: Entre as diversas dificuldades de atenção identificadas em crianças autistas, incluem-se operações seletivas e manutenção dessas atividades [Quill 1997] entendidas como foco; 
V Congresso Brasileiro de Informática na Educação (CBIE 2016)

Anais do XXVII Simpósio Brasileiro de Informática na Educação (SBIE 2016)

- Fixação visual: Crianças neurotípicas em desenvolvimento apresentam maior atratividade por estímulos sociais [Spelke E S 1995], por exemplo, ao olhar para um rosto, a criança olha fixamente para os olhos, em vez de olhar para a boca [Haith et al. 1977]. Contrastando com essa característica, autistas mostram desgosto por essa predisposição social [Dawson et al. 1998];

- Raciocínio lógico: Estudos indicam que crianças a partir de quatro anos possuem capacidade de raciocinar logicamente [English 1993]. Por outro lado, apesar de crianças autistas também apresentarem essa habilidade [Robertson 1993], as mesmas não são atraídas para adotar estratégias lógicas.

Nas últimas décadas, houve um aumento crescente na preocupação, sobretudo, no meio psicológico, psiquiátrico e psicanalítico, com a patologia infantil de forma geral. Segundo o Centro de Prevenção e Controle de Doenças (do inglês, Center of Deseases Control and Prevention - $C D C$ ), atualmente são 2 milhões de autistas nos EUA; no Brasil, foi realizado um estudo na cidade de Atibaia (SP) que registrou um autista para cada 333 crianças e, no mundo, segundo a ONU, estima-se que mais de 70 milhões de pessoas sejam autistas [Junior 2011]. O grande problema do autismo e das psicoses da criança reside num modelo multifacético, fazendo-se necessária uma fonte de procedimentos terapêuticos multi e transdisciplinares que relute ao distúrbio em todas as suas particularidades sindrômicas [Mazet and Lebovici 1991].

A partir dos avanços nas áreas de pesquisa de patologias descritas anteriormente, a integração de softwares para auxílio na confrontação de observações que podem ser úteis do ponto de vista clínico, puderam proporcionar um enriquecimento no tratamento dos pacientes. Kimball e Smith [Kimball and Smith 2007] descreveram os benefícios e desafios no uso de novas tecnologias no tratamento do autismo. Essas técnicas, sozinhas ou em conjunto, podem ser usadas em diferentes aspectos da intervenção terapêutica, através do monitoramento evolutivo das habilidades cognitivas e o próprio uso de uma ferramenta que unificaria várias atividades próprias do auxílio-tratamento, auxiliando os indivíduos, bem como familiares e profissionais que acompanham uma criança com a síndrome autista [Rajendran 2008].

Nesta perspectiva, é proposto no presente artigo um ambiente de jogos interativos para dar suporte ao tratamento de crianças com autismo, denominado TEO (Tratar, Estimular e Orientar). Os jogos presentes no TEO foram desenvolvidos considerando aspectos importantes para estimular a concentração, o foco, a discriminação visual e o raciocínio lógico das crianças. Além disso, buscou-se incorporar ao aplicativo um módulo de coleta de dados do usuário/paciente, permitindo, assim, o monitoramento do progresso da criança à medida que ela utiliza os jogos.

A contribuição central desta trabalho reside no fato de que muitos jogos relacionados ao autismo são desenvolvidos desligados de pesquisa, partindo do princípio de que uma criança autista conseguiria obter os mesmos resultados iniciais que qualquer outra criança sem autismo e emancipada de acompanhamento. Desta forma, o aplicativo TEO, disponível na Google Play ${ }^{1}$, foi desenvolvido através de pesquisas e entrevistas com profissionais das áreas de Psicologia, Terapia Ocupacional e afins, objetivando ainda prover uma forma de mensurar a evolução da criança para um dado jogo a partir de dados coletados à medida que as crianças utilizam os jogos.

O presente artigo está organizado em 6 seções. É detalhado na Seção 2 um levantamento sistemático sobre os jogos disponíveis atualmente para auxiliar no tratamento do autismo. Na Seção 3 é descrita a metodologia utilizada para consolidação de um modelo

\footnotetext{
${ }^{1}$ https://play.google.com/store/apps/details?id=com.Sia. Teo\&hl=ptBR
} 
V Congresso Brasileiro de Informática na Educação (CBIE 2016)

Anais do XXVII Simpósio Brasileiro de Informática na Educação (SBIE 2016)

para criação dos jogos. Na Seção 4 é apresentado o aplicativo TEO bem como sua estrutura e desafios. Os resultados e discussões acerca da experiência do usuário são relatados na Seção 5. E, por fim, as considerações finais sobre o presente trabalho são apresentadas na Seção 6.

\section{Trabalhos relacionados}

Apesar da demasiada quantidade de softwares disponíveis no mercado com diversas finalidades, poucos são os que se encaixam em necessidades específicas e individuais, como no caso do autismo. Ademais, os profissionais que efetuam o acompanhamento podem encontrar grandes dificuldades em aduzir o jogo mais apropriado para cada conjuntura [Sousa and de Castro 2012]. Importantes resultados hoje são obtidos com ajuda de softwares no auxílio ao aprendizado e tratamento do autista, abstraindo metodologias comumente usadas no tratamento [Cerbasi 2012].

Atualmente, dentre os jogos desenvolvidos especificamente para ajudar no tratamento de crianças autistas, pode-se destacar o ComFiM [Ribeiro et al. 2014], o ECHOES [Bernardini et al. 2014] e o ABC Autismo [Farias et al. 2015], apresentados em detalhes nas seções a seguir.

\subsection{ComFiM}

Com o objetivo de promover as habilidades de comunicação em pessoas autistas, através de um jogo multiplayer colaborativo para tablets, o ComFiM estimula a interação do autista com um personagem virtual com o objetivo de familiarizá-lo com a dinâmica do jogo, para logo após incentivar a interação com outro jogador. A partir de uma "fazenda" como cenário, o jogo composto por três níveis de dificuldades conta com a ajuda do Sistema de Comunicação por Figuras (PECS) para que a criança autista possa se comunicar.

Durante a pesquisa acerca do ComFiM verificou-se que a aprovação por meio das crianças demonstrou-se satisfatória, entretanto, uma maior quantidade de crianças com diferentes idades em estudos futuros poderiam ampliar os resultados. Ademais, foi observado que o uso da tecnologia contribuiu para o trabalho em conjunto.

\subsection{ECHOES}

Desenvolvido para ajudar crianças autistas a aprimorar e adquirir habilidades de comunicação social, através de uma tela multi-touch LCD de 42 polegadas com rastreamento de olhar fixo e um agente virtual como parceiro da criança durante o jogo, chamado Andy, para cada criança as atividades são selecionadas por um acompanhante (profissional ou parente) manualmente.

Apesar de não ter havido um aumento significativo nas respostas sociais dos usuários, há evidências de que algumas crianças se beneficiaram da experiência com o software. O software ECHOES apresenta ainda uma das primeiras avaliações realizadas no contexto, contando com diferentes crianças autistas [Bernardini et al. 2014].

\subsection{ABC Autismo}

Recorrendo à metodologia TEACCH (Treatment and Education of Autistic and Related Communication Handicapped Children) [Mesibov et al. 2004], o ABC Autismo é um software desenvolvido na linguagem Java e disponível para tablets com sistema operacional Android. A complexidade do aprendizado seguindo metodologias de tratamento para autismo está abstraído entre os quatro níveis presentes no ABC Autismo, com finalidade de auxiliar no aprendizado da criança de forma adaptada para suas necessidades. 
Ainda que a aceitação do $\mathrm{ABC}$ autismo por parte das crianças não tenha sido muito satisfatória [Farias et al. 2014], acredita-se que em versões posteriores possa haver uma adequação maior por meio de sequências de atividades. É notável que os jogos apresentados demonstram diversos métodos de abstrair conceitos vitais para o tratamento e ensino de autistas, alguns focando em habilidades de comunicação, outros em habilidades cognitivas, porém todos com um único objetivo em comum, o de ser uma ferramenta de auxílio ao tratamento do espectro autista, participando de maneira ativa em todo o processo.

\section{Metodologia}

O ambiente TEO foi proposto como uma suíte de jogos desenvolvida com o propósito de portar para mais de uma plataforma de dispositivos móveis. Desta forma, dada a sua facilidade de concepção e portabilidade, a game engine Unity $^{2}$ foi escolhida com o propósito de desenvolver a suíte. A solução apresentada nesse artigo fundamentou-se em um estudo prévio acerca do autismo que, visando a contribuição de especialistas, resultou em uma metodologia inspirada no desenvolvimento de software iterativo e incremental. A metodologia do presente projeto, descrita a seguir, divide-se em 5 etapas de desenvolvimento: definição, prototipação, validação, implementação e avaliação. Com exceção da fase de avaliação, realizada após a consolidação do aplicativo, todas as demais são iterativas, repetindo-se à medida que novos possíveis incrementos forem identificados.

\subsection{Definição}

A ideia por trás do projeto é recriar os jogos (atividades) comumente empregados no tratamento tradicional e trazê-los para o ambiente digital, como jogos de raciocínio lógicomatemático (ver Figura 1-a), e atividades de vida diária (ver Figura 1-b). Esta fase objetiva identificar novos jogos para o aplicativo bem como seus níveis e desafios. Para tal, foram necessárias pesquisas sobre o autismo, buscando déficits cognitivos comuns, apesar da singularidade apresentada, aspirando através dos mesmos mensurar quais são os princípios focados durante as sessões individuais de um tratamento. Com a parceria da clínica TRATE ${ }^{3}$, foi possível assimilar características importantes para avaliação da criança autista, como acertos, erros, tentativas totais, dificuldades associadas ao movimento com o drag and drop, pedido de auxílio para realizar a atividade e um atraso caracterizado por uma indecisão.

\subsection{Prototipação}

Uma vez definido o escopo da nova atividade, uma versão preliminar é criada com o propósito de sintetizar o que foi discutido na fase anterior e ter em mãos uma versão funcional para a fase de validação. Cada protótipo produzido conta com a lógica básica do jogo, não possui o refinamento final do layout e tem por objetivo apresentar o novo jogo a ser criado.

\subsection{Validação}

Etapa realizada com um grupo de testes, pacientes do TRATE previamente selecionados, sob supervisão de profissionais da clínica TRATE. A avaliação consistiu em reforçar os requisitos colhidos durante a primeira etapa, adaptando-os às dificuldades comuns encontradas nas crianças, como, por exemplo, a dificuldade em trabalhar com operações de

\footnotetext{
${ }^{2}$ Disponível em: https://unity3d.com/pt

${ }^{3}$ Para promover um melhor embasamento para o projeto, foram realizadas reuniões periódicas com a presença de profissionais da clínica Espaço Trate (TRATE), pertencente à prefeitura municipal de Arapiraca-AL, a qual é especializada no tratamento da TEA.
} 


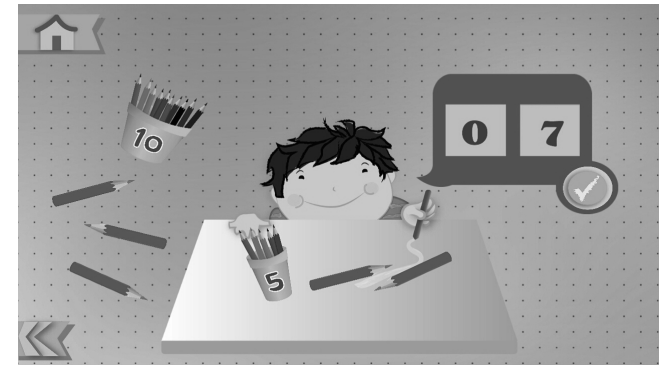

(a) O jogo Simbonúmero desenvolve a habilidade da criança de relacionar números a quantidades.

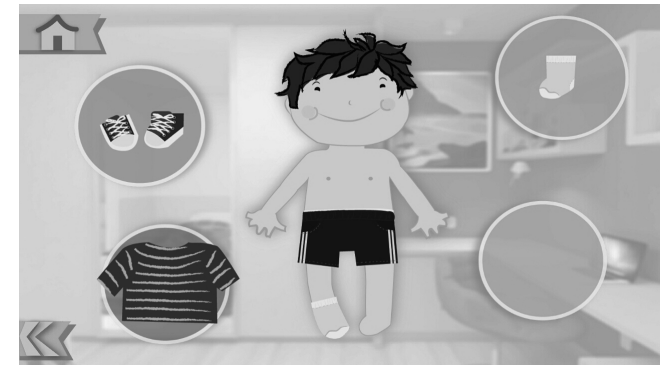

(b) O Jogo Vestir tem por finalidade orientar a criança a como se vestir sozinha.

Figura 1. Exemplos de jogos integrantes da aplicativo TEO.

soma quando há ocorrência do dígito zero em uma das parcelas. Desta forma, juntamente com o feedback fornecido pelos especialistas da TRATE, buscou-se desenvolver o aplicativo de modo que este possa corresponder às condições da criança a fim de torná-lo adaptável ao ambiente terapêutico.

\subsection{Implementação}

A etapa de implementação compreende o processo de refinação do protótipo criado, desenvolvendo um novo jogo a partir dos requisitos coletados durante a definição e resultados obtidos na validação. Elementos atrativos foram utilizados visando atrair o interesse da criança, tais como efeitos sonoros que indicam diversão e imagens coloridas de objetos conhecidos. A fim de validar o novo artefato produzido, a etapa de validação é novamente realizada e o ciclo se repete até não restarem novos aprimoramentos.

\subsection{Avaliação}

A avaliação realizada sobre o aplicativo TEO deu-se por meio de métricas de usabilidade, visando validar ou encontrar falhas que possam comprometer as atividades da criança. Foram selecionados 5 profissionais especializados (psicólogos e terapeutas) para testarem e avaliarem o aplicativo. Um questionário, descrito na Tabela 1, composto de 10 questões seguindo a escala Likert foi aplicado buscando extrair informações que possam contribuir para a avaliação. No geral, buscou-se identificar dificuldades em manusear o aplicativo (problemas de usabilidade), a utilidade deste como uma ferramenta auxiliar ao tratamento e a relevância dos dados coletados, assim as questões foram separadas em 3 grupos distintos: A, B e C.

Tabela 1: Questionário aplicado na etapa de avaliação.

\begin{tabular}{|l|l|}
\hline Questões & Alternativas \\
\hline \hline $\begin{array}{l}\text { A1. Como você classifica a facilidade em encontrar os itens na } \\
\text { tela (botões de seleção etc.)? }\end{array}$ & \multirow{2}{*}{ (a) Muito ruim. } \\
\cline { 1 - 1 } A2. Como você classifica o tamanho e a qualidade das imagens? & \\
\cline { 1 - 1 } A3. Como você classifica as fontes e tamanhos das letras? & (b) Ruim. \\
\cline { 1 - 1 } A4. Como você classifica a sequência de telas? & \\
\hline A5. Como você classifica a navegação do aplicativo? & \\
\hline
\end{tabular}


V Congresso Brasileiro de Informática na Educação (CBIE 2016)

Anais do XXVII Simpósio Brasileiro de Informática na Educação (SBIE 2016)

Tabela 1 - continuação da página anterior

\begin{tabular}{|c|c|}
\hline Questões & Alternativas \\
\hline $\begin{array}{l}\text { B1. Como você classifica o nível de confiança do aplicativo } \\
\text { como auxiliar no tratamento? }\end{array}$ & \multirow{5}{*}{$\begin{array}{l}\text { (c) Moderado. } \\
\text { (d) Ótimo. } \\
\text { (e) Excelente. }\end{array}$} \\
\hline $\begin{array}{l}\text { B2. Como você classifica o valor do aplicativo para o trata- } \\
\text { mento? }\end{array}$ & \\
\hline $\begin{array}{l}\text { C1. Como você classifica as informações coletadas do paciente } \\
\text { pelo aplicativo? }\end{array}$ & \\
\hline C2. Como você classifica a utilidade dessas informações? & \\
\hline $\begin{array}{l}\text { C3. Como você classifica a importância de mostrar as } \\
\text { informações coletadas ao término do jogo? }\end{array}$ & \\
\hline
\end{tabular}

\section{O Ambiente de Jogos TEO}

Após a descoberta e diagnóstico do autismo, surgem diversas questões relacionadas ao tratamento, já que ainda não existe uma cura comprovada. Um tratamento único não existe, uma vez que o transtorno apresenta uma singularidade em cada indivíduo. Por meio da clínica TRATE e com a ajuda de médicos especializados envolvidos no processo e o total apoio dos pais, pode-se obter uma melhora nas habilidades sociais e comunicativas, além de reduzir os sintomas do autismo.

A proposta envolvida no aplicativo TEO diz respeito ao auxílio no tratamento de crianças com autismo a partir do uso do tablet, garantindo uma abordagem multidisciplinar para que alcance seu potencial. Faz-se uso da tecnologia na busca por estimular as crianças através de atividades presentes em um aplicativo para Android, para que sejam trabalhadas suas habilidades cognitivas, fomentando seu raciocínio lógico e outras habilidades diversas. Através dos conceitos trabalhados nos jogos, foi possível dividi-los por categorias, como é possível observar na Figura 2-b, sendo elas:

1. Associação: com o objetivo de fomentar habilidades cognitivas, propõe-se uma atividade simples de condução e assimilação de objetos, aprimorando o processamento e reconhecimento de formas e cores;

2. Raciocínio lógico-matemático: disponibiliza jogos que buscam desenvolver a ideia de unidades, dezenas, soma e subtração através da assimilação com objetos do cenário;

3. Quebra-cabeça: jogo tradicional com objetivo de estimular as habilidades cognitivas da criança autista como distância e tentativa-erro através de imagens ilustrativas com temática infantil;

4. Memória: jogo tradicional com objetivo de estimular a concentração, capacidade de observação e atenção do autista por meio de um conjunto de figuras separadas por contexto;

5. Atividades de vida diária: também chamada de AVD, esta categoria proporciona jogos capazes de estimular a criança a realizar tarefas pessoais do dia a dia, tais como autocuidados e atividades cotidianas;

6. Localização: ensina, por meio de perguntas, a localização de objetos, tais como partes do corpo, visando aumentar o vocabulário da criança e buscando trabalhar o aspecto da comunicação.

O conteúdo visual do aplicativo TEO foi concebido com a finalidade de criar um ambiente confortável para uma criança com o TEA e, ao mesmo tempo, atrativo e divertido para quaisquer outras crianças. Ayres [Ayres 1972] explica que a integração sensorial 
V Congresso Brasileiro de Informática na Educação (CBIE 2016)

Anais do XXVII Simpósio Brasileiro de Informática na Educação (SBIE 2016)

com o equilíbrio entre a quantidade e qualidade de estímulos recebidos por um indivíduo é a resposta dada pelo mesmo, tendo início nos primeiros meses de vida. O desenvolvimento infantil está diretamente relacionado com integração sensorial, que não ocorre de maneira adequada em crianças com autismo. Dessa forma, a percepção que a criança autista tem do ambiente ocorre diferente de crianças neurotípicas, assim o sistema visual, entre outros sentidos dessas crianças apresentam certas particularidades que ainda é difícil de mensurar.

Através de pesquisas para propor a integração sensorial de crianças autistas [Ayres 1972], foi pretendido para o ambiente TEO uma maior diversidade de cores suaves bem como intensas. As cores intensas porém, são bastante pontuais, usadas para chamar atenção, não sobrecarregando a visão do usuário. Os ícones presentes no aplicativo (ver Figura 2) foram planejados especificamente para o mesmo, criados com base no Sistema de Comunicação por Figuras (PECS) [Charlop-Christy et al. 2002], amplamente usado por terapeutas e pais como incentivo à comunicação da criança autista.

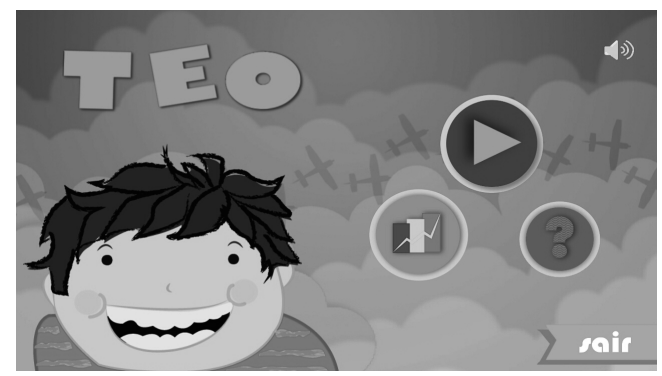

(a) Menu principal apresentando o mascote Teo.

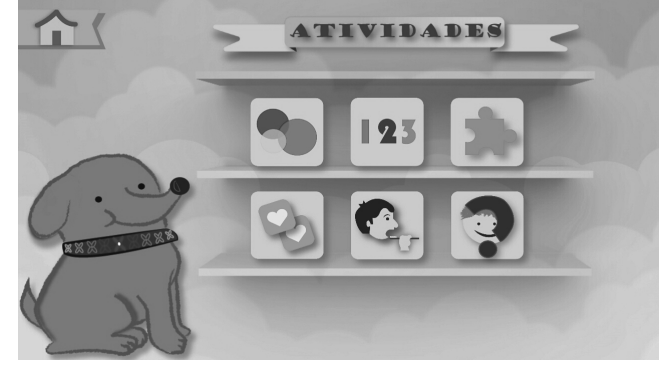

(b) Suíte de jogos com 6 categorias.

Figura 2. Predominância de botões grandes e intuitivos nas telas principais do aplicativo.

Concomitantemente à proposta dos jogos em auxílio ao processo de tratamento de crianças autistas, tendo como ponto de vista um serviço adicional à avaliação feita pelo profissional médico, a ideia de inserir técnicas de mineração de dados e de inteligência artificial foi considerada para o presente projeto. Através do monitoramento da jogabilidade de cada usuário, objetiva-se coletar características peculiares dos mesmos, como forma de modelar informações que possibilitem classificar seus perfis informalmente e recomendar jogos com base nas principais dificuldades encontradas.

Dados como quantidade de erros e acertos, tempo para completar uma partida, entre outros, melhor descrito em [Filho et al. 2015], são coletados e disponibilizados no formato .csv, na memória local do dispositivo, para que possam ser objetos de avaliação para outras áreas de estudo bem como para os profissionais da psicologia e terapeutas ocupacionais.

\section{Resultados e discussão}

Os resultados apresentados (ver Figura 3) descrevem a inspeção realizada por profissionais responsáveis pelo tratamento, direcionando a análise aos dados coletados e à interface do usuário através de um questionário, descrito na Seção 3.5. A partir do questionário, foi possível analisar a experiência do usuário em contato com o software. É importante mencionar que o aplicativo TEO foi desenvolvido visando reforçar a colaboração entre o terapeuta e paciente, assim, o usuário não se restringe apenas à criança.

O primeiro grupo de questões, cujo prefixo é A, tem como finalidade investigar dificuldades encontradas pelo usuário em usufruir das funcionalidades fornecidas pelo 


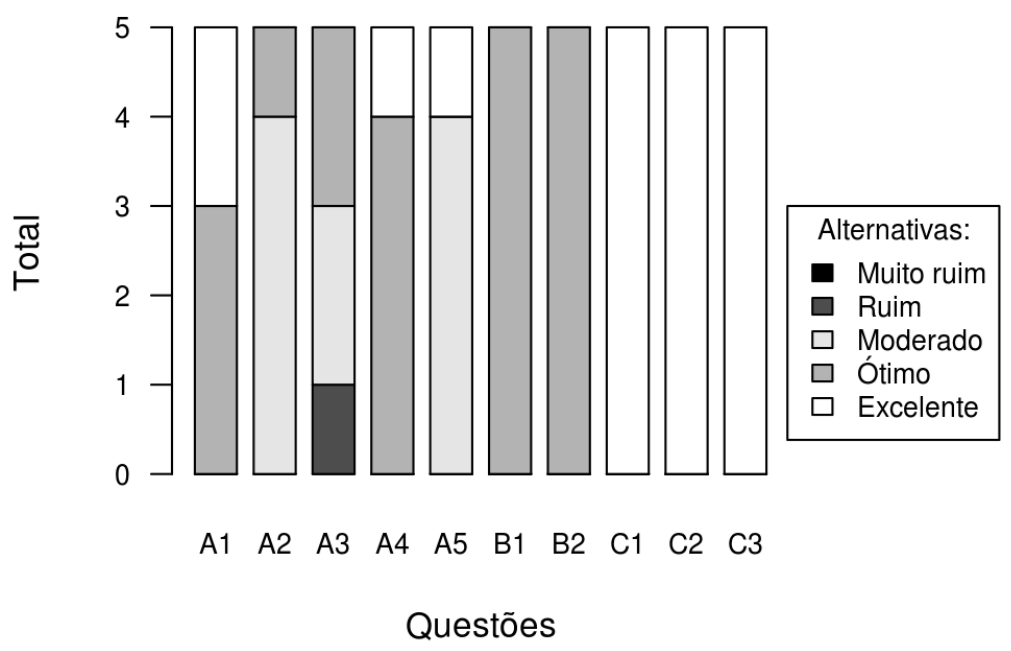

Figura 3. Resultado do questionário

aplicativo, inclusive as atividades. Dos 3 grupos de questões, o grupo A teve a maior divergência entre as respostas. Na questão A1, os entrevistados não relataram problemas quanto à disposição dos itens na tela, $60 \%$ dos participantes responderam como "ótimo", enquanto que os outros $40 \%$ optaram por "excelente" como resposta.

Visando acrescentar recomendações ergométricas ao nosso estudo, as questões A2 e A3 foram propostas intencionando identificar possíveis problemas em assimilar elementos textuais e não-textuais dentro do jogo. A partir dos resultados obtidos da questão A2, foi possível avaliar o tamanho e qualidade das imagens. Nesta perspectiva, $80 \%$ dos entrevistados responderam "moderado" e $20 \%$ como "ótimo". Durante a inspeção, alguns usuários alegaram uma certa dificuldade ao lerem os textos no aplicativo. Na questão A3, 40\% dos entrevistados avaliaram como "ótimo", 40\% avaliaram como "moderado" e $20 \%$ como "ruim". Todos os entrevistados utilizaram o mesmo dispositivo. Ambos os problemas acentuam-se quando tablets com resoluções relativamente menores são utilizados para o aplicativo. Neste caso, pressupõe-se a necessidade de adaptar o layout de modo a atender uma gama maior de dispositivos, uma vez que o sistema Android não se limita apenas aos tablets - muitos usuários de smartphones também têm realizado a instalação do aplicativo.

As questões A4 e A5, apesar de similares, tratam de assuntos diferentes. A questão A4 avalia se a transição de uma tela para outra está consistente. Nesta perspectiva, $80 \%$ dos entrevistados avaliaram a sequência de telas como "ótima", 20\% apontou como "excelente". A questão A5 tem por finalidade avaliar a organização hierárquica das telas, desde o menu inicial até a tela dos jogos. Para tal, $80 \%$ dos entrevistados avaliaram como "moderado" e $20 \%$ como "excelente". A predominância da avaliação moderada para essa questão sugere um desconforto ao acessar as telas do aplicativo.

O grupo B apresenta questões que tentam captar a opinião dos especialistas quanto à importância do aplicativo como uma ferramenta auxiliar ao tratamento. Para as duas questões do grupo, todos os entrevistados concordaram em "ótimo" como resposta. As respostas das questões $\mathrm{C} 1, \mathrm{C} 2$ e C 3 evidenciam a importância dos dados coletados durante a partida, uma vez que todos os entrevistados responderam "excelente" para as 3 questões. Dentre os aplicativos citados nos trabalhos relacionados, nenhum oferecia meios de acompanhar o progresso do paciente ao término das atividades. A coleta de dados é, portanto, 
V Congresso Brasileiro de Informática na Educação (CBIE 2016)

Anais do XXVII Simpósio Brasileiro de Informática na Educação (SBIE 2016)

um importante diferencial que destaca a relevância do aplicativo TEO.

O aplicativo TEO encontra-se disponível gratuitamente em lojas virtuais, contabilizando mais de 5 mil downloads em diversos países, um número bastante expressivo que reflete a importância e a necessidade de investimentos em novas metodologias para o tratamento do autismo. O sucesso motivacional conquistado pelos ambientes digitais aliado ao fácil acesso, tornam o aplicativo TEO uma ferramenta de suma relevância social, mas que ainda encontra-se em processo de validação. No futuro, espera-se avaliar o aplicativo com um número maior de especialistas e com as crianças, obtendo, deste modo, resultados concretos capazes de mensurar com mais exatidão a influência do aplicativo no contexto terapêutico.

\section{Considerações Finais}

No presente artigo foi proposto um ambiente de jogos para dispositivos móveis cujo objetivo é dar suporte ao tratamento de crianças com autismo. Tal ambiente dispõe-se de uma suíte de jogos com diversas categorias, além de disponibilizar informações pertinentes à jogabilidade da criança, como meta de que se possa trabalhar vários conceitos fundamentais para o tratamento. Ademais, com a portabilidade para diversas plataformas, $o$ aplicativo tende a ter um custo menor de implantação por clínicas e familiares, uma vez que pode ser embarcado em diferentes dispositivos móveis de baixo custo.

Uma vez que o principal objetivo é uma constante melhora da criança autista, trabalhando ainda em suas deficiências específicas, os dados coletados ao longo dos jogos poderão servir como métricas para a futura implementação dos sistemas de recomendação de tratamento. Justifica-se esta abordagem pois um mapa discreto das métricas auxiliará a identificação dos perfis de usuários de maneira automatizada e poderá acompanhar a evolução dos jogadores para proporcionar um tratamento de maior qualidade.

Por fim, com o objetivo de interagir com um cenário ainda tão desconhecido, como é o universo autista, a pesquisa realizada no desenvolvimento do aplicativo continua de maneira a se aprimorar cada vez mais, bem como as propostas em adicionar outros recursos que auxiliem no tratamento e interação das tecnologias atuais.

\section{Referências}

Ayres, A. J. (1972). Sensory integration and learning disorders. Western Psychological Services.

Bernardini, S., Porayska-Pomsta, K., e Smith, T. J. (2014). Echoes: An intelligent serious game for fostering social communication in children with autism. Information Sciences, 264:41-60.

Cerbasi, J. (2012). Computer programs and apps for children with autism. http://www. foxnews.com/health/2012/04/13/ computer-programs-and-apps-for-children-with-autism/.

Acessado em: 30 de maio de 2016.

Charlop-Christy, M. H., Carpenter, M., Le, L., LeBlanc, L. A., e Kellet, K. (2002). Using the picture exchange communication system (PECS) with children with autism: assessment of PECS acquisition, speech, social-communicative behavior, and problem behavior. Journal of applied behavior analysis, 35(3):213-31.

Dawson, G., Meltzoff, A. N., Osterling, J., Rinaldi, J., e Brown, E. (1998). Children with autism fail to orient to naturally occurring social stimuli. Journal of Autism and Developmental Disorders, 28(6):479-485. 
V Congresso Brasileiro de Informática na Educação (CBIE 2016)

Anais do XXVII Simpósio Brasileiro de Informática na Educação (SBIE 2016)

English, L. (1993). Evidence for deductive reasoning: Implicit versus explicit recognition of syllogistic structure. British Journal of Developmental Psychology, 11(4):391-409.

Farias, E., Cunha, M., e Souza, J. W. (2015). ABC Autismo - Uma Aplicação Mobile para Auxiliar no Processo Alfabetizador de Crianças com Autismo. In Anais dos Workshops do IV Congresso Brasileiro de Informática na Educação (CBIE), page 232.

Farias, E. B., Silva, L. W. C., e Cunha, M. X. C. (2014). ABC AUTISMO : Um aplicativo móvel para auxiliar na alfabetização de crianças com autismo baseado no Programa TEACCH. In X Simpósio Brasileiro de Sistemas de Informação, pages 458-469.

Filho, D. L. S. O., Moura, D. L. L., Silva, A. J. G., da Silva Queiroz, F., Cavalcante, R. C., e de Sales, T. B. M. (2015). Um Novo Conceito de Sistemas Interativos para Autistas com Prospecção de Dados e Sistemas de Recomendação. In XIII Workshop de Educação e Informática da Escola Regional de Computação Bahia - Alagoas - Sergipe (Erbase).

Haith, M., Bergman, T., e Moore, M. (1977). Eye contact and face scanning in early infancy. Science, 198(4319):853-855.

Joseph, R. M., Keehn, B., Connolly, C., Wolfe, J. M., e Horowitz, T. S. (2009). Why is visual search superior in autism spectrum disorder? Developmental Science, 12(6):1083-1096.

Junior, P. (2011). Brasil é destaque no dia mundial do autismo. http: //www . revistaautismo.com.br/diamundial2011. Acessado em: 30 de maio de 2016.

Kanner, L. (1943). Austistic disturbances of affective contact. Nervous Child., 2:217-250.

Kimball, J. W. e Smith, K. (2007). Crossing the bridge: From best practices to software packages. Focus on Autism and Other Developmental Disabilities, 22, 131-134, 22(2):131-134.

Mazet e Lebovici (1991). Autismo e Psicoses da Criança. Artes Médicas.

Mesibov, G. B., Shea, V. S., e Schopler, E. (2004). The TEACCH approach to autism spectrum disorders. Springer.

Quill, E. K. (1997). Instructional considerations for young children with autism: The rationale for visually cued instruction. Autism and Developmental Disorders, 27(6):697714.

Rajendran, G. (2008). Enhancing and accelerating the pace of autism research and treatment. Focus on Autism and Other Developmental Disabilities, 23(2):125-129.

Ribeiro, P. C., d. Araujo, B. B. P. L., e Raposo, A. (2014). Comfim: A cooperative serious game to encourage the development of communicative skills between children with autism. In 2014 Brazilian Symposium on Computer Games and Digital Entertainment, pages $148-157$.

Robertson, J. R. (1993). Verbal analogical reasoning in severely learning disabled children. unpublished manuscript. University of Kent.

Sousa, F. R. M. e de Castro, T. H. C. (2012). Worldtour: Towards an adaptive software to support children with autism in tour planning. In 2012 IEEE 36th International Conference on Computer Software and Applications.

Spelke E S, Phillips A, W. A. L. (1995). Infants' knowledge of object motion and human action. Oxford University Press, Oxford. 